\title{
Colchicine-Induced Rhabdomyolysis and Possible Amiodarone Interaction
}

\author{
Colchicine-Induced Rhabdomyolysis
}

\author{
Chaker Ben Salem ${ }^{1}$, Jaballah Sakhri ${ }^{2}$, Neila Fathallah ${ }^{1}$, Besma Trimech ${ }^{3}$, Houssem Hmouda ${ }^{4}$, \\ Bouraoui Kamel $^{1}$
}

${ }^{1}$ Department of Clinical Pharmacology, Faculty of Medicine of Sousse, Sousse, Tunisia; ${ }^{2}$ Department of Surgery, Farhat Hached Hospital, Sousse, Tunisia; ${ }^{3}$ Department of Cardiology, Farhat Hached Hospital, Sousse, Tunisia; ${ }^{4}$ Medical Intensive Care Unit, Sahloul Hospital, Sousse, Tunisia.

Email: bensalem.c@gmail.com

Received August 10 ${ }^{\text {th }}, 2010$; revised August 25 $5^{\text {th }}, 2010$; accepted September $5^{\text {th }}, 2010$.

\begin{abstract}
Objective: To report a case of drug interaction leading to rhabdomyolysis. Case Summary: A 65-year old woman suffering from chronic atrial fibrillation was treated with amiodarone and acenocoumarol. Two weeks after administration of conventional dosage of colchicine for pericarditis, the patient developed rhabdomyolysis. colchicine-induced rhabdomyolysis was suspected. Colchicine was stopped and the patient underwent supportive therapy. Clinical symptoms improved rapidly. Discussion: Colchicine-induced neuromuscular toxicity and rhabdomyolysis have been reported with chronic treatment in therapeutic doses. Concomitant use of several drugs with colchicine may potentiate the development of myopathy. In our case, a co-administration of colchicine, a well known substrate of cytochrome P450 $3 A 4$ and P-glycoprotein, and amiodarone had possibly precipitated rhabdomyolysis. Amiodarone may increase colchicine toxicity by a dual mechanism. Amiodarone inhibits P-glycoprotein which may theoretically result in increased intracellular colchicine concentrations and decreased hepatic and renal excretion of the drug. Conclusion: Amiodarone may potentiate the development of colchicine-induced rhabdomyolysis.
\end{abstract}

Keywords: Colchicine, Rhabdomyolysis, Amiodarone, Interaction

\section{Introduction}

Colchicine is an anti-inflammatory drug widely used in the treatment of a large panel of inflammatory diseases and particularly gout. Colchicine may induce many side effects, principally gastrointestinal adverse effects including abdominal pain, vomiting, and diarrhea. It may cause much more severe reactions such as bone marrow depression, myoneuropathy and myopathy. Rhabdomyolysis is a rare and lifethreatening adverse effect of colchicine. We report a case of rhabdomyolysis possibly induced by colchicine and concomitant use of amiodarone.

\section{Case Report}

A 65-year old woman suffering from chronic atrial fibrillation treated with amiodarone ( $200 \mathrm{mg}$ daily; 5 days per week) and acenocoumarol ( $0.25 \mathrm{mg}$ daily). She also received captopril $(25 \mathrm{mg}$ daily); furosemide $(40 \mathrm{mg}$ daily); molsidomine (75 mg daily) and spironolactone
(50 mg daily) for ischemic cardiomyopathy. Colchicine therapy (1 $\mathrm{mg}$ daily) has been started two weeks before admission for pericarditis. She was admitted to cardiology department for management of over-anticoagulation by acenocoumarol manifested by epistaxis and melena. On admission, the patient was afebrile. The relevant physical findings were atrial fibrillation without signs of acute decompensated heart failure. The patient also reported myalgia and diffuse muscle weakness. Both sensation and coordination were intact. She had no recent viral illness and she denied any change from her normal level of activity. No alcohol ingestion, illicit drugs, or trauma were present. There were no signs of liver insufficiency or for thyroid disease.

Laboratory tests revealed the following: international normalized ratio (INR) at $6.5 \mathrm{UI} / 1$, creatine kinase (CK) at $5780 \mathrm{UI} / \mathrm{L}$ (normal range: $40-150 \mathrm{U} / \mathrm{L}$ ) and aldolase at $3700 \mathrm{UI} / \mathrm{L}$ (normal range: 06-16 UI/L). Blood cell count, renal function, liver enzymes, potassium and troponin 
serum levels were within normal range. Viral tests did not disclose any viral infection. Based on clinical and biological findings, colchicine-induced rhabdomyolysis was suspected. Muscle biopsy was not performed because of high INR. Colchicine was stopped and the patient underwent supportive therapy. The other drugs were given continuously. Clinical symptoms improved rapidly with a progressive decrease in $\mathrm{CK}$ and aldolase levels. Few days later, CK level decreased to $384 \mathrm{UI} / \mathrm{L}$, and aldolase level to $100 \mathrm{UI} / \mathrm{L}$. However, the patient died from ventricular fibrillation.

\section{Discussion}

Colchicine-induced neuromuscular toxicity and rhabdomyolysis have been reported with chronic treatment in therapeutic doses. Patients with renal dysfunction and elderly patients, even those with normal renal and hepatic function, are at increased risk. Concomitant use of atorvastatin, simvastatin, pravastatin, lovastatin, fluvastatin, gemfibrozil, fenofibrate, fenofibric acid, or benzafibrate (themselves associated with myotoxicity) or cyclosporine may potentiate the development of myopathy [1-4].

Colchicine is a well known substrate of cytochrome P450 3A4 (CYP3A4) in the liver and gastrointestinal (GI) tract, along with P-glycoprotein efflux pumps (P-gp) in the GI tract. Potent CYP3A4 inhibitors such as clarithromycin, cyclosporine, diltiazem, erythromycin, grapefruit juice, itraconazole, ketoconazole, and verapamil may increase colchicine levels and the subsequent risk of toxicity [5]. Many CYP3A4 inhibitors also inhibit P-gp, which could further increase this effect.

Amiodarone is a well known CYP3A4 and P-gp inhibitor [6,7]. This inhibition is primarily because of its active metabolite, desethylamiodarone, which noncompetitively inhibits $3 \mathrm{~A} 4$. Thus, Amiodarone co-administration may increase colchicine toxicity by a dual mechanism. Amiodarone inhibits P-glycoprotein which may theoretically result in increased intracellular colchicine concentrations and decreased hepatic and renal excretion of the drug. Amiodarone may also interact with CYP3A4 to decrease the hepatic elimination of colchicine. Several drugs increase the potential for colchicine toxicity via dual modulation of CYP3A4 and P-gp [8]. These include the macrolide antibiotics erythromycin and clarithro-mycin, and the statins (lovastatin, simvastatin, atorvastatin).

Based on the Naranjo probability scale, it is probable that colchicine caused this patient's rhabdomyolysis, and the Horn drug interaction probability scale indicates a possible interaction between colchicine and amiodarone $[9,10]$. Our case highlights a previously unknown drug interaction. Rhabdomyolysis developed few days after starting standard dose of colchicine in a patient without renal insufficiency.

The FDA recently reviewed the safety of oral colchicine [5]. New drug interactions have been identified with this agent. Oral colchicine was linked to 169 cases of fatal toxicity. One-hundred seventeen cases occured in patients taking therapeutic doses, and over half of these 117 cases involved concomitant use of clarithromycin.

Colchicine-induced rhabdomyolysis is an acute lifethreatening disease. The main goal of treatment is to stop muscle destruction. The rapid withdrawal of the drug is crucial. Once colchicine is stopped, the symptoms generally resolve within 1 week to several months [5].

The exact mechanism of colchicine-induced myoneuropathy is still unclear. Colchicine affects microtubular cell function, it may cause disruption of axonal transport and organelle trafficking in both nerve and muscle cells at the bases of clinical deficits. Electromyography shows fibrillations, positive sharp waves, and low-amplitude distal motor and sensory potentials. If performed, muscle biopsy shows characteristic vacuolar myopathy with no associated necrosis. In another side, the over-anticoagulation by acenocoumarol manifested by epistaxis and melena (reason for admission) may be precipitated by amiodarone co-administration. The potentiation of acenocoumarol anticoagulant effect by amiodarone is well established. In our patient, concomitant medications such as captopril, furosemide, molsidomine and spironolactone seem to not interfere with colchicine. These drugs are not known to be CYP3A4 or P-gp inhibitor.

At present, colchicine has been recommended by the 2004 European guidelines on the management of pericardial diseases for acute (class IIa) and recurrent pericarditis (class I), but its use is still unlabeled and informed consent is required for prescription [11].

The indication of treatment with colchicine should be carefully considered especially in poly-medicated patients. Patients should be informed that muscle pain or weakness may occur with colchicine alone or when it is used with certain other drugs such as amiodarone. Patients should be educated to report symptoms of myopathy immediately to physicians who may decide to discontinue colchicine treatment.

\section{Conclusions}

Our case suggests a possible interaction between colchicine and amiodarone that appears theoretically possible but not yet confirmed. We recommend that further in vivo studies be completed to definitively identify the mechanism of the interaction amiodarone-colchicine.

\section{REFERENCES}

[1] W. C. Hsu, W. H. Chen, M. T. Chang and H. C. Chiu, 
"Colchicine-Induced Acute Myopathy in a Patient with Concomitant Use of Simvastatin," Clinical Neuropharmacology, Vol. 25, No. 5, September-October 2002, pp.266-8.

[2] G. Alayli, K. Cengiz, F. Cantürk, D. Durmus, Y. Akyol, E.B. Menekse, "Acute Myopathy in a Patient with Concomitant Use of Pravastatin and Colchicines" Annals of Pharmacotherapy, Vol. 39, No. 5, July-August 2005, pp. 1358-1361.

[3] I. Akdag, A. Ersoy, S. Kahvecioglu, M. Gullulu and K. Dilek, "Acute Colchicine Intoxication during Clarithromycin Administration in Patients with Chronic Renal Failure" Journal of Nephrology, Vol. 19, No. 4, JulyAugust 2006, pp. 515-517.

[4] I. F. Hung, A. K. Wu, V. C. Cheng, B. S. Tang, K. W. To, C. K. Yeung, P. C. Woo, S. K. Lau, B. M. Cheung and K. Y. Yuen, "Fatal Interaction between Clarithromycin and Colchicine in Patients with Renal Insufficiency: A Retrospective Study," Clinical Infectious Diseases, Vol. 41, No. 3, August 2005, pp. 291-300.

[5] http://www.accessdata.fda.gov/drugsatfda_docs/label/200 9/022351lbl.pdf

[6] M. Katoh, M. Nakajima, H. Yamazaki and H. T. Yokoi,
"Inhibitory Effects of CYP3A4 Substrates and their Metabolites on P-Glycoprotein-Mediated Transport," European Journal of Pharmaceutical Sciences, Vol. 12, No. 4, February 2001, pp. 505-513.

[7] W. Yamreudeewong, M. DeBisschop, L. G. Martin, D. L Lower, "Potentially Significant Drug Interactions of Class III Antiarrhythmic Drugs," Drug Safety, Vol. 26, No. 6, June 2003, pp. 421-438.

[8] E. Niel and J. M. Scherrmann, "Colchicine Today," Joint Bone Spine, Vol. 73, No. 6, 2006, pp. 672-678.

[9] C. A. Naranjo, U. Busto, E. M. Sellers, P. Sandor, I. Ruiz, E. A. Roberts, E. Janecek, C. Domecq and D. J. Greenblatt, "A Method for Estimating the Probability of Adverse Drug Reactions," Clinical Pharmacology Therapeutics, Vol. 30, No. 2, August 1981, pp. 239-245.

[10] J. R. Horn, P. D. Hansten and L. N. Chan, "Proposal for a New Tool to Evaluate Drug Interaction Cases," Annals of Pharmacotherapy, Vol. 41, No. 4, April 2007, pp. 674 680.

[11] M. Imazio, R. Trinchero and Y. Adler, "Colchicine for the Treatment of Pericarditis," Future Cardiology, Vol. 4, No. 6, November 2008, pp. 599-607. 